\title{
The extended uncertainty principle inspires the Rényi entropy
}

\author{
H. Moradpour ${ }^{1 *}$, C. Corda ${ }^{2 \dagger}$, A. H. Ziaie ${ }^{1 \ddagger}$, S. Ghaffari ${ }^{1 \S}$ \\ 1 Research Institute for Astronomy and Astrophysics of Maragha (RIAAM), Maragha 55134-441, Iran \\ 2 International Institute for Applicable Mathematics and Information Sciences, \\ B. M. Birla Science Centre, Adarshnagar, Hyderabad 500063, India
}

\begin{abstract}
We use the extended uncertainty principle (EUP) in order to obtain the Rényi entropy for a black hole $(\mathrm{BH})$. The result implies that the non-extensivity parameter, appeared in the Rényi entropy formalism, may be evaluated from the considerations which lead to EUP. It is also shown that, for excited BHs, the Rényi entropy is a function of the $\mathrm{BH}$ principal quantum number, i.e. the $\mathrm{BH}$ quantum excited state. Temperature and heat capacity of the excited BHs are also investigated addressing two phases while only one of them can be stable. At this situation, whereas entropy is vanished, temperature may take a non-zero positive minimum value, depending on the value of the non-extensivity parameter. The evaporation time of excited $\mathrm{BH}$ has also been studied.
\end{abstract}

\section{INTRODUCTION}

In one hand, uncertainty principle inspires Bekenstein entropy, and indeed, various generalized uncertainty principles (GUP) add different modifications to Bekenstein entropy [1 5]. On the other hand, Bekenstein entropy is a non-extensive entropy measure, a property which motivates some physicists to consider it as a suitable candidate for the Tsallis entropy in calculating the Rényi entropy [6-10]. In fact, due to the long-range nature of gravity [11], the use of generalized entropy formalisms such as those introduced by Tsallis [12] and Rényi 13] has recently been taken into consideration [6$10,14,15]$.

A long-range interacting system with $W$ discrete states while each state $i$ has probability $P_{i}$ may follow a power probability distribution instead of the ordinary distribution [11 13]. Working with $G=c=k_{B}=\hbar=\frac{1}{4 \pi \epsilon_{0}}=1$ (Planck units), where $k_{B}$ denotes the Boltzmann constant, the Tsallis entropy of such system is defined as [12]

$$
S_{T}=\frac{1}{1-q} \sum_{i=1}^{W}\left(P_{i}^{q}-P_{i}\right),
$$

where $q$ is an unknown parameter [11]. It is worthwhile mentioning that one can reach $S_{T}=\frac{A}{4}$, where $A$ denotes the horizon of system (boundary), by applying the Tsallis entropy definition (11) to the gravitational systems [16]. This result is in agreement with the cosmological studies in which authors assumed $S_{T}=\frac{A}{4}$ in calculating Rényi entropy [6-10] written as [11]

$$
\mathcal{S}=\frac{1}{\delta} \ln \left(1+\delta S_{T}\right)
$$

in which $\delta \equiv 1-q$. In addition to the successes of this entropy in describing cosmos [6 8, 10], it can also be combined with the Verlinde's hypothesis [17] to give us a theoretical basis for the MOND theory and its modifications [9]. In this paper, we are going to show that EUP can also lead to the emergence of Rényi entropy. In addition, relation between $\delta$ (and thus $q$ ) and the quantum mechanical parameter appeared in EUP is also derived. After getting the mentioned aim in the next section, we study some thermodynamic properties of excited BHs meeting Rényi entropy in the third section. The last section is devoted to summary and concluding remarks.

\section{FROM EUP TO RÉNYI ENTROPY}

In the framework of the high energy physics, such as quantum gravity, various GUP and EUP are derived 1, 18 21], which can generally be written as [18]

$$
\Delta x \Delta p \geq\left[1+\beta(\Delta x)^{2}+\eta(\Delta p)^{2}+\gamma\right] .
$$

Here, $\gamma$ is positive and depends on the expectation values of $p$ and $x$ [18, 22]. Bearing the fact that the minimum uncertainty is obtainable for $\gamma=0$ in mind [22], we consider the $\eta=\gamma=0$ case which leads to EUP written as [1, $18,21,23$.

$$
\Delta x \Delta p \geq\left[1+\frac{\beta \pi}{4}(\Delta x)^{2}\right],
$$

where $\beta$ is positive and independent of the values of $\Delta x$ and $\Delta p[18]$. The non-zero minimal values of $\Delta x$ and $\Delta p$, called $\Delta x_{0}$ and $\Delta p_{0}$, respectively, are obtainable whenever $\beta>0$ [18]. The above EUP affects the early universe thermodynamics 22], and in general, there are deep connections between EUP and GUP and $i$ ) the dispersion relation [25, 26], ii) the Chandrasekhar and Jeans limits and the dark energy problem [23, 24]. More studies on the outcomes of employing GUP in various branches of physics can also be found in $[1,5,27-33]$.

\footnotetext{
*h.moradpour@riaam.ac.ir

†cordac.galilei@gmail.com

‡ah.ziaie@riaam.ac.ir

$\S$ sh.ghaffari@riaam.ac.ir
} 
Whenever the EUP (4) is valid, one can write $\Delta E \approx$ $\Delta p$ for the uncertainty of the particle energy $(\Delta E)[3-$ 5, 30 32, leading to

$$
\Delta E \geq \frac{1}{\Delta x}\left[1+\frac{\beta \pi}{4}(\Delta x)^{2}\right]
$$

In $\mathrm{BH}$ physics, whenever a $\mathrm{BH}$ with area $A$ absorbs or emits a quantum particle with energy $E$ and size $R$, then the changes in the $\mathrm{BH}$ area follows the $\Delta A \geq 8 \pi E R$ relation [28 30]. Since the size of a quantum particle cannot be less than the uncertainty in its position [29, 30, 34], one reaches $\Delta A_{\min } \geq 8 \pi E \Delta x$ for a quantum particle [5, 29, 30]. Combining this result with Eq. (5), we reach at

$$
\Delta A_{\min } \geq 8 \pi\left[1+\frac{\beta \pi}{4}(\Delta x)^{2}\right]
$$

As it has been argued in Refs. [5, 28 30], one can write $(\Delta x)^{2} \approx \frac{A}{\pi}$ and insert it in Eq. (6) to obtain

$$
\Delta A_{\min } \simeq 8 \pi \lambda\left[1+\frac{\beta}{4} A\right]
$$

where $\lambda$ is an unknown coefficient fixed later [5].

Therefore, $\Delta A_{\min }$ is the minimum changes in the boundary $A$ whenever EUP (4) is valid. It is also obvious to assume that the corresponding entropy changes is also minimum and equal to one bit of information $\Delta S_{\min }=b=\ln 2[5,17,34]$. The above argument motivates us to write

$$
\frac{d S}{d A}=\frac{\Delta S_{\min }}{\Delta A_{\min }}=\frac{b}{8 \pi \lambda\left[1+\frac{\beta}{4} A\right]},
$$

leading to

$$
S=\frac{b}{2 \pi \lambda \beta} \ln \left[1+\frac{\beta}{4} A\right]
$$

In the limit of $\beta \rightarrow 0$, the Bekenstein entropy $\left(\frac{A}{4}\right)$ should be recovered [1 $15,34,35]$ which yields

$$
\lambda=\frac{b}{2 \pi}
$$

whereby we get

$$
S=\mathcal{S}, \quad \delta=\beta .
$$

Thus, one can realize that $i$ ) EUP may allow us to employ the Rényi entropy, and in this situation, ii) the EUP parameter $\beta$ determines the value of the non-extensivity parameter $\delta$. Finally, it is also worthwhile mentioning that the value of $\lambda$ obtained in Eq. (10) is the same as that of the previous work by other authors [5] in which the $\beta=\gamma=0$ case has been studied.

\section{APPLICATIONS TO THE EXCITED BHS}

For excited BHs, i.e. the BHs which emitted a large amount of Hawking quanta, the recent Bohr-like approach to BH quantum physics in 36 -38] permits to write the Bekenstein entropy in terms of the $\mathrm{BH}$ quantum level as 38

$$
\frac{A}{4}=4 \pi\left(M^{2}-\frac{n}{2}\right),
$$

where $M$ is original $\mathrm{BH}$ mass and $n$ is the $\mathrm{BH}$ principal quantum number if the $\mathrm{BH}$ is seen as gravitational atom, see e.g., 36 38. We indeed recall that, the intuitive but general belief [36 38]: the $\mathrm{BHs}$ result in highly excited states representing both the Hydrogen atom and the quasi - thermal emission in quantum gravity, has been shown to be correct, because the Schwarzschild BH results in somewhat similar to the historical semi-classical hydrogen atom introduced by Bohr in 1913, see 36 38 for more details. Thus, by using Eqs. (2) and (11), the Rényi entropy becomes function of the $\mathrm{BH}$ principal quantum number, i.e., the $\mathrm{BH}$ excited state, given as

$$
S=\beta^{-1} \ln \left[1+4 \beta \pi\left(M^{2}-\frac{n}{2}\right)\right]
$$

Accepting the $E=M$ relation and bearing the $T=\frac{\partial E}{\partial S}$ relation in mind, one reaches

$$
T=\frac{1+4 \beta \pi\left(M^{2}-\frac{n}{2}\right)}{8 \pi M},
$$

for temperature of the Hawking radiation in this formalism. As a check, the temperature $T_{B}=\frac{1}{8 \pi M}$, obtained by using the Bekenstein entropy, is also recovered at the appropriate limit $\beta \rightarrow 0$. In this manner, for $n \rightarrow n_{\max }=2 M^{2}$ [38], we have $S \rightarrow 0$ and $T \rightarrow T_{B}$ independent of the value of $\beta$. Moreover, the heat capacity evaluated as

$$
C=\frac{\partial M}{\partial T}=\frac{8 \pi M^{2}}{2 \beta \pi\left(2 M^{2}+n\right)-1},
$$

includes a singularity at $n=n_{\max }$ whenever $\beta=\frac{1}{8 \pi M^{2}} \equiv$ $\beta_{0}$. For this critical value of $n$, we have $C>0(C<0)$ for $\beta>\beta_{0}\left(\beta<\beta_{0}\right)$, and in neighboring of this point one can write $C \sim \frac{1}{\beta-\beta_{0}}$. This means that the $\beta>\beta_{0}$ $\left(\beta<\beta_{0}\right)$ phase can be stable (unstable) [39 41]. For the critical value $\beta_{0}$, one can write (14) as

$$
T=\frac{T_{B}}{2}\left[3-\frac{n}{2 M^{2}}\right],
$$

indicating $T>T_{B}$ for $n<n_{\max }$ which means that $T_{B}$ is the minimum possible temperature at this situation. 
More studies on the non-excited BHs $(n=0)$ as well as their thermodynamics in the framework of the Rényi entropy can also be found in [39, 42, 43].

Now, let us look at the radiation of excited $\mathrm{BH}$ as a black body radiation, and write [40, 42]

$$
\frac{d M}{d t}=-16 \pi M^{2} \sigma T^{4}(M)
$$

where $\sigma$ denotes the Stefan-Boltzman constant, and additionally, we assumed that the $r=2 M$ relation is still valid [38]. Therefore, by using Eq. (14), the time that a $\mathrm{BH}$ needs to lose its mass $M$ can be evaluated as

$$
t=-\frac{4(4 \pi)^{3}}{\sigma} \int_{M}^{0} \frac{M^{2} d M}{\left(1+4 \beta \pi\left(M^{2}-\frac{n}{2}\right)\right)^{4}},
$$

leading to

$$
\begin{aligned}
& \tilde{t}=\frac{3 \tan ^{-1}\left[\frac{\sqrt{8 \pi \beta} M}{\sqrt{2-4 \pi \beta n}}\right]}{\sqrt{1-2 \pi \beta n}}+\frac{1}{\left(4 \pi \beta\left(n-2 M^{2}\right)-2\right)^{3}}( \\
& 2 \sqrt{4 \pi \beta} M\left((4 \pi \beta)^{2}\left[3 n^{2}+16 n M^{2}-12 M^{4}\right]+12\right. \\
& \left.\left.-16 \pi \beta\left(3 n+8 M^{2}\right)\right)\right)
\end{aligned}
$$

where $\tilde{t} \equiv 3 \sigma t\left(\frac{\beta}{4 \pi}\right)^{\frac{3}{2}}$. Whenever $\beta=0$ or even $n=n_{\max }$, one obtains

$$
t=\frac{4(4 \pi)^{3}}{3 \sigma} M^{3}
$$

the evaporation time of a Schwarzschild BH [42]. The reason is clear, for both addressed cases, Eq. (14) reduces to the ordinary temperature of Schwarzschild BH [38]. It is also worthwhile mentioning some similarities and differences between the properties of an excited Schwarzschild BH in the Bekenstein and Rényi entropy formalisms. i) Both the Bekenstein (12) and Rényi (13) entropies are vanished for $n=n_{\max }$, $\left.i i\right)$ in the framework of the Bekenstein entropy, temperature is always independent of $n$, and $i i i$ ) heat capacity is always negative, independent of the value of $n$, in the regime of the Bekenstein entropy (the $\beta=0$ limit of (15)), while $C$ can be positive depending on the values of $n$ and $\beta$. For example, whereas $n=n_{\max }$, we have $C>0$ if $\beta>\beta_{0}$.

\section{SUMMARY AND CONCLUDING REMARKS}

The Rényi entropy for a BH has been obtained through EUP. By using the recent Bohr-like approach to $\mathrm{BH}$ quantum physics, we have also shown that, for excited $\mathrm{BHs}$, the Rényi entropy is a function of the $\mathrm{BH}$ principal quantum number, i.e. the $\mathrm{BH}$ quantum excited state. Some thermodynamic properties of excited $\mathrm{BH}$ meeting Rényi entropy have also been addressed. The results show that although there are two phases at $n \rightarrow n_{\max }$, only the phase with $\beta>\beta_{0}$ can be stable. Moreover, whenever $n \rightarrow n_{\max }$, then entropy is vanished, and temperature takes its non-zero possible minimum value $\left(T_{B}\right)$ if $\beta=\beta_{0}$.

Evaporation time of the excited BHs has also been studied. Although we addressed the $n=n_{\max }$ case in our study, in reality, the maximum possible value of $n$, namely $N_{\max }$, may be limited as $N_{\max }=n_{\max }-1$ whenever the Planck mass and the Planck distance are approached [38, 44]. In this manner, non of the Bekenstein and Rényi entropies are vanished when the maximum value of $n$ is taken by the excited $\mathrm{BH}$. It leads to the interesting results in the Rényi entropy framework, in agreement with the third law of thermodynamics, $i$ ) based on Eq. (13), entropy takes its minimum value which is nonzero, and $i i$ ) from Eq. (14), the minimum of temperature can be positive or even greater than $T_{B}$ depending on the value of $\beta$.

\section{Acknowledgment}

The work of H. Moradpour has been supported financially by Research Institute for Astronomy \& Astrophysics of Maragha (RIAAM).
[1] F. J. Wang, Y. X. Gui, Y. Zhang, Gen Relativ Gravit 41, 2381 (2009).

[2] B. Majumder, Astrophys. Space. Sci. 336, 331 (2011).

[3] A. F. Ali, A. Tawfik, AHEP 2013, 126528 (2013).

[4] A. F. Ali, Phys. Lett. B 732, 335 (2014).

[5] A. Awada, A. F. Ali, JHEP 06, 093 (2014).

[6] N. Komatsu, Eur. Phys. J. C 77, 229 (2017).

[7] H. Moradpour, A. Bonilla, E. M. C. Abreu, J. A. Neto, Phys. Rev. D 96, 123504 (2017).

[8] H. Moradpour, et al. Eur. Phys. J. C 78, 829 (2018).

[9] H. Moradpour, A. Sheykhi, C. Corda, I. G. Salako, Phys.
Lett. B 783, 82 (2018).

[10] H. Moradpour, A. H. Ziaei, S. Ghaffari, V. B. Bezerra, under review in EPJC.

[11] M. Masi, Phys. Lett. A 338, 217 (2005).

[12] C. Tsallis, J. Stat. Phys. 52, 479 (1988).

[13] A. Rényi, Probability Theory (North-Holland, Amsterdam, 1970).

[14] M. Tavayef, A. Sheykhi, K. Bamba, H. Moradpour, Phys. Lett. B 781, 195 (2018).

[15] A. Sayahian Jahromi et al., Phys. Lett. B 780, 21 (2018).

[16] A. Majhi, Phys. Lett. B 775, 32 (2017). 
[17] E. P. Verlinde, JHEP 1104, 029 (2011).

[18] A. Kempf, G. Mangano, R. B. Mann, Phys. Rev. D 52, 1108 (1995).

[19] B. Bolen, M. Cavaglia, Gen. Relativ. Gravit. 37, 1255 (2005).

[20] M. I. Park, Phys. Lett. B 659, 698 (2008).

[21] C. Bambi, F. R. Urban, Class. Quantum Grav. 25, 095006 (2008).

[22] K. Norazi, B. Fazlpour, Gen. Relativ. Gravit. 38, 1661 (2006).

[23] Y. C. Ong, Y. Yao, Phys. Rev. D 98, 126018 (2018).

[24] H. Moradpour et al., Accepted By Mon. Not. Roy. Astro. Soc. (2019).

[25] S. Hossenfelder, Class. Quantum. Grav. 23, 1815 (2006).

[26] K. Nozari, A. S. Sefiedgar, Phys. Lett. B 635, 156 (2006).

[27] L. Xiang, et al., Ann. Phys. 396, 334 (2018).

[28] A. J. M. Medved and E. C. Vagenas, Phys. Rev. D 70, 124021 (2004).

[29] B. Majumder, Phys. Lett. B 703, 402 (2011).

[30] M. Cavaglia, S. Das, Class. Quant. Grav. 21, 4511 (2004).

[31] F. Scardigli, Nuovo Cimento B 110, 1029 (1995).
[32] R. J. Adler, P. Chen, D. I. Santiago, Gen. Relativ. Gravit. 33, 2101 (2001).

[33] P. Pedram, Phys. Lett. B 718, 638 (2012).

[34] J. D. Bekenstein, Phys. Rev. D 7, 2333 (1973).

[35] M. Srednicki, Phys. Rev. Lett. 71, 666 (1993).

[36] C. Corda, Class. Quantum Gravity 32, 195007 (2015).

[37] Y. Heydarzade, H. Hadi, C. Corda, F. Darabi, Phys. Lett. B 776, 457 (2018).

[38] C. Corda, Adv. High Energy Phys. 867601 (2015).

[39] V. G. Czinner, H. Iguchi, Phys. Lett. B 752, 306 (2016).

[40] S. Ghaffari, et al., arXiv:1901.01506.

[41] H. B. Callen, Thermodynamics and Introduction to Thermostatics (New York: John Wiley and Sons, 1985); H. B. Callen, Thermodynamics (J. Wiley, NY, 1960).

[42] T. S. Biró, V. G. Czinner, Phys. Lett. B 726, 861 (2013).

[43] A. Bialas, W. Czyz, Euro. Phys. Lett. 83, 60009 (2008).

[44] R. J. Adler, P. Chen, D. I. Santiago, Gen. Rel. Grav. 3, 2101-2108 (2001, Third Award in the Gravity Research Foundation Essay Competition). 